\title{
Changes in the redox state of Escherichia coli cells during phosphate starvation
}

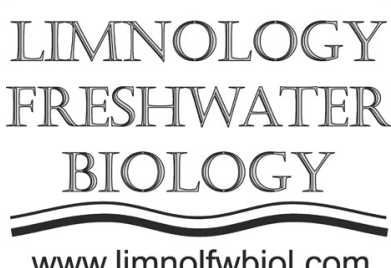

www.limnolfwbiol.com

\author{
Smirnova G.V.*, Tyulenev A.V., Muzyka N.G., Ushakov V.Y., Oktyabrsky O.N. \\ Institute of Ecology and Genetics of Microorganisms, Perm Federal Research Center, Russian Academy of Sciences, ul. Goleva 13, Perm, \\ 614081, Russia
}

\begin{abstract}
Redox regulation of cellular processes is one of the most important factors providing metabolic reprogramming when bacteria adapt to environmental changes. The aim of this work was to study changes in the redox status of glutathione in E. coli cells during phosphate starvation. We showed that the transition of $E$. coli from growth to phosphate starvation is accompanied by $\mathrm{H}_{2} \mathrm{~S}$ production, export of free cysteine to the medium, and an increase in the concentration of intracellular glutathione. An increase in the level of reduced glutathione (GSH) in the absence of changes in the concentration of oxidized glutathione (GSSG) led to an increase in the ratio of GSH/GSSG inside wild-type cells and all studied mutants in the components of thiol redox systems. High redox status of the cells was maintained during two days of starvation. Intensive oxidation of GSH during phosphate starvation occurred only in the simultaneous absence of glutathione reductase and thioredoxin reductase. We suggest that the observed changes in the level of thiols and $\mathrm{H}_{2} \mathrm{~S}$ are largely aimed at maintaining cysteine homeostasis and preventing oxidative stress.
\end{abstract}

Keywords: glutathione, $\mathrm{H}_{2} \mathrm{~S}$, cellular redox systems, phosphate starvation, Escherichia coli

\section{Introduction}

The life cycle of intestinal bacteria includes the stage of entry into aquatic ecosystems, where they encounter a cardinal change in the living conditions (physicochemical composition of the medium, $\mathrm{pH}$, temperature, nutrient limitation). Adaptation to these changes requires interconnected rearrangements of all metabolic pathways of bacterial cells. Among the regulatory signals providing this metabolic reprogramming, an important role is played by redox regulation based on changes in the concentration of reactive oxygen species and low molecular weight thiols, which are able to interact with sensitive SHgroups of enzymes and regulatory factors, affecting their activity (Oktyabrsky and Smirnova, 2007). E. coli has two intracellular thiol redox systems: a glutathione redox system including glutathione (GSH), glutathione reductase (GOR) and glutaredoxins, and a thioredoxin redox system including thioredoxins (TrxA and TrxC) and thioredoxin reductase (TrxB). E. coli cells contain millimolar concentrations of GSH, which participates in maintaining intracellular cysteine homeostasis and serves as the main cytoplasmic redox buffer (Smirnova et al., 2019). The aim of this work was to study the changes in the redox state of glutathione in wild-type $E$. coli and mutants in various components of thiol redox systems during phosphate starvation.

\section{Material and methods}

A parental strain of $E$. coli BW25113 (wt) and single-knockout mutants JW2663 ( $\Delta g s h A)$, JW3467 ( $\triangle$ gor), JW5856 ( $\Delta$ trxA), JW0871 ( $\Delta$ trxB) were from the Keio collection. The double mutants gshAtrxA and gortrx $B$ were created by transduction with phage P1. Bacteria were grown on MOPS medium with $8.5 \mathrm{mM}$ glucose and $4 \mathrm{mM}$ phosphate in 250-ml flasks with shaking $(150 \mathrm{rpm})$ at $37^{\circ} \mathrm{C}$. In phosphate starvation experiments, a culture grown to $\mathrm{OD}_{600}$ of 0.6 was centrifuged and transferred to MOPS with glucose, but without phosphate. A starving culture was maintained under aerobic conditions for 48 hours. Changes in dissolved oxygen, redox potential of the medium (Eh), and extracellular potassium and sulfide concentrations were monitored in real time using a Clark electrode, a platinum electrode, and selective electrodes for $\mathrm{K}^{+}$ and $\mathrm{S}^{2}$. In addition, $\mathrm{H}_{2} \mathrm{~S}$ was recorded in the gas phase using paper strips soaked in lead acetate. GSH, GSSG and cysteine inside and outside the cells were measured by sensitive spectrophotometric methods. Membrane potential was determined using a $\operatorname{DiBAC}_{4}(3)$ fluorescent dye and a Leica DM2000 microscope.

\section{Results}

The transition from growth to phosphate starvation in all strains was accompanied by a sharp 
inhibition of growth, respiration, and partial reversible release of $\mathrm{K}^{+}$from the cells. During the first hour of starvation, there was a decrease in the level of glutathione in the medium and its 3-fold increase inside the cells. The concentration of intracellular GSSG $\left(\right.$ GSSG $_{\text {in }}$ ) did not change significantly, with the exception of the gortrx $B$ mutant, in which it increased 1.5 times. The level of extracellular cysteine increased 2.4 times in the wild-type culture and 5.1 times in the gshA mutant. A characteristic feature of the transition process was an increase in the concentration of sulfide in the medium, where it was recorded by platinum and sulfide electrodes as an abrupt decrease in potential, the amplitude of which corresponded to a sulfide concentration of $225 \pm 5 \mathrm{nM}$. The sulfide concentration in the gortrx $B$ mutant was 2 times lower than that of the parent. The gsh $A$ mutant generated sulfide for a longer period than all other strains. The results of measuring $\mathrm{H}_{2} \mathrm{~S}$ in the gas phase corresponded to the data obtained using electrodes. Changes in all studied parameters were reversible. The addition of phosphate led to the rapid resumption of growth and respiration, accelerated consumption of $\mathrm{K}^{+}$and the rapid release of GSH from the cells into the medium. Prolonged (for 2 days) phosphate starvation did not lead to a decrease in the survival and membrane potential of bacteria. The concentration of intracellular $\mathrm{GSH}\left(\mathrm{GSH}_{\mathrm{in}}\right)$ and the ratio of $\mathrm{GSH}_{\text {in }} / \mathrm{GSSG}_{\text {in }}$ were maintained at a higher level than in the growing culture in all strains, with the exception of the gor and gortr $x B$ mutants. The level of GSSG $_{\text {in }}$, which increased sharply during the first hour of starvation in the gortr $x B$ mutant, returned to its initial value after 24 hours due to the excretion of GSSG in the medium, where its 4-fold increase was observed. The concentration of reduced glutathione in the medium in all strains decreased to almost zero.

\section{Discussion}

Our studies showed that the transition of $E$. coli from growth to phosphate starvation is accompanied by
$\mathrm{H}_{2} \mathrm{~S}$ production, release of free cysteine from the cells into the medium, and an increase in the concentration of intracellular GSH. Previously, similar changes were observed with inhibition of protein synthesis in $E$. coli in response to the addition of valine or chloramphenicol (Smirnova et al., 2019). These changes can be considered as a non-specific reaction to starvation stress and a way to regulate the level of intracellular cysteine, which is able to potentiate oxidative stress, under conditions of sharp inhibition of protein synthesis.

\section{Conclusions}

Thus, when adapting of $E$. coli to phosphate starvation, significant changes in the concentration and redox state of low molecular weight thiols are observed, which are largely aimed at maintaining cysteine homeostasis and reducing the risk of oxidative stress. Intensive oxidation of GSH during phosphate starvation occurs only with the simultaneous absence of GOR and TrxB. GSSG formed under these conditions is excreted into the medium.

\section{Acknowledgements}

This work was carried out in accordance with state assignment AAAA-A19-119112290009-1 and supported by grants from the Russian Foundation for Basic Research 19-04-00888, the President of the Russian Federation for young scientists MK-420.2020.4 and Norpexal Foundation.

\section{References}

Oktyabrsky O.N., Smirnova G.V. 2007. Redox regulation of cellular functions. Biochemistry (Moscow) 72: 132-145. DOI: $10.1134 /$ s0006297907020022

Smirnova G.V., Tyulenev A.V., Bezmaternykh K.V. et al., 2019. Cysteine homeostasis under inhibition of protein synthesis in Escherichia coli cells. Amino Acids 51: 1577-1592. DOI: $10.1007 /$ s00726-019-02795-2 